\title{
Critical transport and critical scattering in fluids
}

\author{
R.Folk \\ Institute for Theoretical Physics, University of Linz, Austria
}

Received March 1, 2000

We consider the critical properties of fluids induced by the critical fluctuations in the order parameter. The theory describes the crossover from the analytic background behaviour to the universal asymptotic behaviour of several dynamic quantities.

Key words: gas-liquid phase transition, critical dynamics, light scattering, transport coefficients

PACS: 05.70.Jk, 64.60.Ht, 64.70.Fx, 64.70.Ja

\section{Introduction}

Collective effects dominate near the phase transition while there arises a critical behaviour quite different from the behaviour further away from the critical point. These critical phenomena are seen in the experiments in a more or less wide region round the critical point. E.g., the thermal conductivity of a pure liquid diverges strongly, with an exponent of $\mathcal{O}(1)$, whereas the shear viscosity diverges weakly, with an exponent of $\mathcal{O}(0.1)$, as a function of the relative temperature distance from the critical point with some power law in the region asymptotically near the critical temperature $T_{\mathrm{c}}$. Outside this region, a normal analytic behaviour is observed. A delicate point is the strength of the critical behaviour compared to the noncritical or background behaviour and determining the background values of the transport properties. Depending on this strength, both types of behaviour can be separated with more or less accuracy. In a series of papers, the crossover behaviour from criticality to the background behaviour has been studied $[1,2]$ and extended to mixtures [3]. The goal of these studies was the description of all dynamical quantities within the formalism of the field theoretic formulation of renormalization group (RG) theory [4]. One advantage of the nonasymptotic RG-theory is that the calculations lead directly to the complete value (without the analytic temperature dependence) of the transport coefficients in the background and no separation of the fluctuation contribution as in the mode coupling theory has to be performed [5].

An important issue in the description of the critical behaviour is the appropriate choice of the collective variables [7] connected to the so-called order parameter (OP). 
In statics near the liquid-gas critical point in pure liquids these are the deviations of the density from the critical density, in dynamics according to model $\mathrm{H}$ [8] it is the entropy density fluctuation $\Delta \sigma$, and we introduce the order parameter field $\phi_{0}$ given by

$$
\phi_{0}(x)=\sqrt{N_{\mathrm{A}}}(\triangle \sigma(x)-\langle\triangle \sigma(x)\rangle) .
$$

In addition, one has to consider the tranverse momentum current $\boldsymbol{j}_{t}$ which is dynamically coupled to the OP. The Hamiltonian describing thermodynamic equilibrium is

$$
H=\int \mathrm{d}^{d} x\left\{\frac{1}{2} \stackrel{o}{\tau} \phi_{0}^{2}(x)+\frac{1}{2}\left(\nabla \phi_{0}(x)\right)^{2}+\frac{\stackrel{o}{\tilde{u}}}{4 !} \phi_{0}^{4}(x)+\frac{1}{2} a_{j} \boldsymbol{j}_{t}^{2}(x)\right\}
$$

and the dynamic equations are

$$
\begin{aligned}
\frac{\partial \phi_{0}}{\partial t}= & \stackrel{o}{\Gamma} \nabla^{2} \frac{\delta H}{\delta \phi_{0}}-\stackrel{o}{g}\left(\nabla \phi_{0}\right) \frac{\delta H}{\delta \boldsymbol{j}}+\Theta_{\phi} \\
\frac{\partial \boldsymbol{j}_{t}}{\partial t}= & \stackrel{o}{\lambda_{t}} \nabla^{2} \frac{\delta H}{\delta \boldsymbol{j}_{t}}+\stackrel{o}{g} \mathcal{T}\left\{\left(\boldsymbol{\nabla} \phi_{0}\right) \frac{\delta H}{\delta \phi_{0}}\right\} \\
& -\stackrel{o}{g} \mathcal{T}\left\{\sum_{k}\left[j_{k} \boldsymbol{\nabla} \frac{\delta H}{\delta j_{k}}+\nabla_{k} \boldsymbol{j} \frac{\delta H}{\delta j_{k}}\right]\right\}+\boldsymbol{\Theta}_{t} .
\end{aligned}
$$

$\mathcal{T}$ is the projector to the direction of the transverse momentum density, which corresponds to a projection orthogonal to the wave vector in Fourier space. In the fast fluctuating forces $\Theta_{i}(x, t)(i=\phi, t)$ memory effects are irrelevant and their Gaussian spectrum fulfils the Einstein relations

$$
\left\langle\Theta_{i}(x, t) \Theta_{j}\left(x^{\prime}, t^{\prime}\right)\right\rangle=2 L_{i j}(x) \delta\left(t-t^{\prime}\right) \delta\left(x-x^{\prime}\right),
$$

where the matrix $\left[L_{i j}\right]$ of the diffusive modes is given by

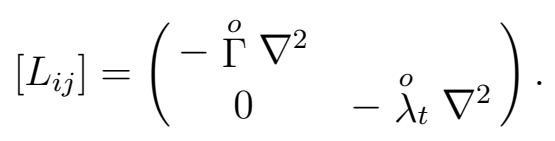

The nonrenormalized mode coupling is defined as $\stackrel{o}{g}=R T / \sqrt{N_{\mathrm{A}}}$ with the gas constant $R$ and the Avogadro number $N_{\mathrm{A}}$.

Within this model all static and dynamic critical properties can be calculated. One obtains especially the correlation functions, and from their half width the characteristic frequencies. In the hydrodynamic region the transport coefficients as functions of temperature result from these frequencies [1]. Our theory yields all these dynamical quantities as functions of the measurable correlation length $\xi$ and the dynamical parameters entering the model equations (3) and (4). The most important is the renormalized mode coupling $f_{t}$ (its nonrenormalized counterpart is $\stackrel{o}{g} / \sqrt{\stackrel{o o}{\Gamma} \lambda_{t}}$ ). It's value for a certain temperature, wave vector or frequency, is determined by the solution (see equation (9) below) of a renormalization group equation and an appropriate matching condition. 


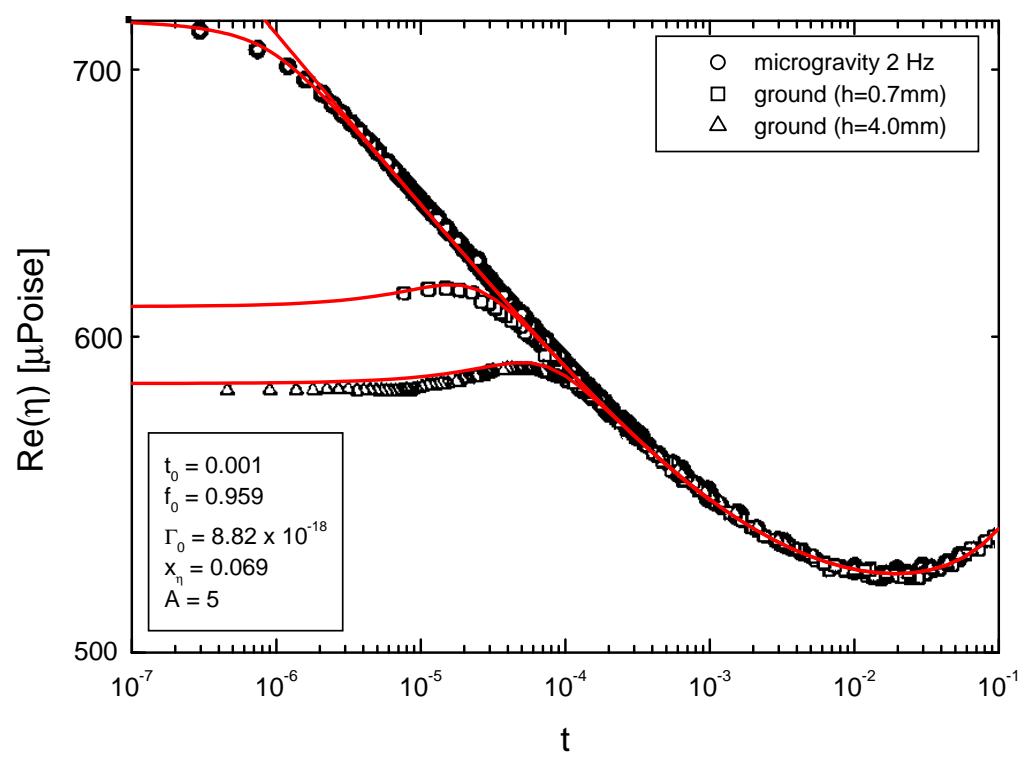

Figure 1. Shear viscosity of xenon [9] with and without gravity compared with RG theory.

\section{Shear viscosity}

The temperature and density dependent shear viscosity at zero frequency calculated within model $\mathrm{H}$ reads [2]

$$
\bar{\eta}(t, \Delta \rho)=\bar{\eta}_{0} \frac{1-f_{t}^{2}(t, \Delta \rho) / 36}{1-f_{0}^{2} / 36}\left(\frac{f_{0}^{2} \xi(t, \Delta \rho)}{f_{t}^{2}(t, \Delta \rho) \xi\left(t_{0}\right)}\right)^{x_{\eta}} \equiv \bar{\eta}_{0} \exp \left(x_{\eta} H(t, \Delta \rho)\right),
$$

with the amplitude

$$
\bar{\eta}_{0}=\frac{k_{\mathrm{B}} T}{4 \pi} \frac{\xi\left(t_{0}\right)}{f_{0}^{2} \Gamma_{0}}\left(1-\frac{f_{0}^{2}}{36}\right)
$$

and the mode coupling

$$
f_{t}^{2}(t, \Delta \rho)=\frac{24}{19}\left[1+\frac{\xi\left(t_{0}\right)}{\xi(t, \Delta \rho)}\left(\frac{24}{19 f_{0}^{2}}-1\right)\right]^{-1}
$$

The dependence on the relative temperature distance from the critical temperature $T_{\mathrm{c}}$ and the relative density distance from the critical density $\rho_{\mathrm{c}}$ enters via the correlation length $\xi(t, \Delta \rho)$ which is obtained from the equations

$$
\begin{gathered}
t=\frac{T-T_{\mathrm{c}}}{T_{\mathrm{c}}}=\left(1-b^{2} \theta^{2}\right) r, \\
\Delta \rho=\frac{\rho-\rho_{\mathrm{c}}}{\rho_{\mathrm{c}}}=k\left(\theta+c \theta^{3}\right) r^{\beta}, \\
\xi(t, \Delta \rho)=\xi_{0}\left(1+0.16 \theta^{2}\right) r^{-\nu}=\xi_{0} t^{-\nu}\left(1+0.16 \theta^{2}\right)\left(1-b^{2} \theta^{2}\right)^{\nu},
\end{gathered}
$$

of the cubic model [6]. 

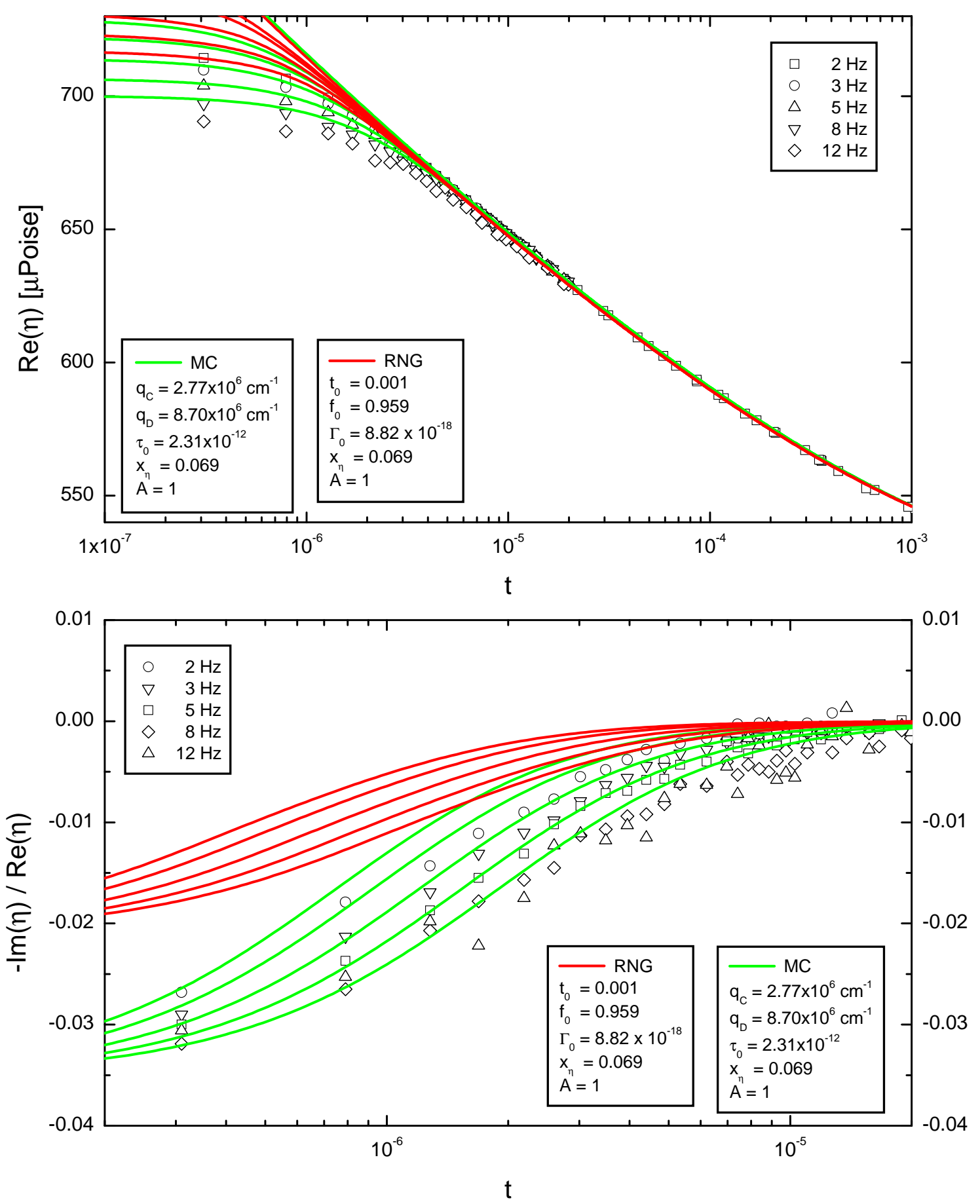

Figure 2. Comparison of the mode coupling theory [10] (light gray) and the RG theory (dark). Data from [9]. 
It is necessary to know the density dependence to calculate the gravity effects on the shear viscosity measurements on earth. Because of the finite height of the cell, a density gradient over the cell height is caused by gravity and depending on the positions of the oscillating disks by which the shear viscosity is measured a mean value of shear viscosities at different densities is measured. Since the gravity field is conjugated to the order parameter, the gradient over the cell increases when one approaches the critical temperature and this drives the shear viscosity away from the critical point at $t=\Delta \rho=0$. Therefore it reaches a finite value instead of diverging near the critical point. This is demonstrated in figure 1 where two measurements on earth in different cells and the measurements on board the "Discovery" [9] are shown together with our calculations.

In the low gravity experiment, the frequency dependence of the shear viscosity can be observed. This is not possible on earth since the gravity effects already cover up the frequency effects. The frequency dependence of the shear viscosity was also calculated in one loop order $[1,2]$ and we compare our result with the low gravity experiments [9] and mode coupling theory [10] in figure 2. No agreement is found in both theories. Introducing a phenomenological parameter into the frequency scale [9] agreement can be achieved in mode coupling theory for both the real part and the ratio of the real part to the imaginary part of the viscosity. This also holds for the RG-result for the real part of the viscosity (with a value different [11] from the mode coupling theory). The ratio of the real part to the imaginary part of the viscosity cannot be improved by this procedure since its limiting value at $T_{\mathrm{c}}$ is given by the universal one loop order result $[11]\left(f_{t}^{* 2}=24 / 19\right)$

$$
\lim _{T \rightarrow T_{\mathrm{c}}} \frac{\Im(\eta)}{\Re(\eta)}=\frac{f_{t}^{* 2}}{96} \frac{\pi}{2}\left[1-\frac{f_{t}^{* 2}}{96}\{3 \ln (1 / 4)-1 / 3\}\right]^{-1} \simeq 0.0195 .
$$

Within the mode coupling theory of $[10,9]$ the value

$$
\lim _{T \rightarrow T_{\mathrm{c}}} \frac{\Im(\eta)}{\Re(\eta)}=\tan \frac{\pi x_{\eta}}{2\left(3+x_{\eta}\right)} \simeq 0.0353
$$

is obtained (the experimental value $x_{\eta}=0.069$ of the shear viscosity exponent is used) and seems to be in agreement with the data. As a result, one might conclude that the one loop theory is not sufficient to describe the critical frequency dependence of the shear viscosity. A complete two loop calculation is in preparation [12].

\section{Thermal diffusivity}

For a complete description of the dynamic critical behaviour it is also necessary to consider the thermal conductivity or thermal diffusivity. Once the values of the nondynamical universal parameters such as $\Gamma_{0}$ and $f_{0}$ are determined, all quantities can be calculated from one theory. E.g., the thermal diffusivity reads

$$
D_{T}(t, \Delta \rho)=D_{0} \frac{\xi^{2}\left(t_{0}\right)}{\xi^{2}(t, \Delta \rho)} \frac{\left(1-\frac{f_{t}^{2}(t, \Delta \rho)}{16}\right)}{\left(1-\frac{f_{0}^{2}}{16}\right)}\left(\frac{f_{0}^{2} \xi(t . \Delta \rho)}{f_{t}^{2}(t, \Delta \rho) \xi\left(t_{0}\right)}\right)^{x_{\kappa}}
$$



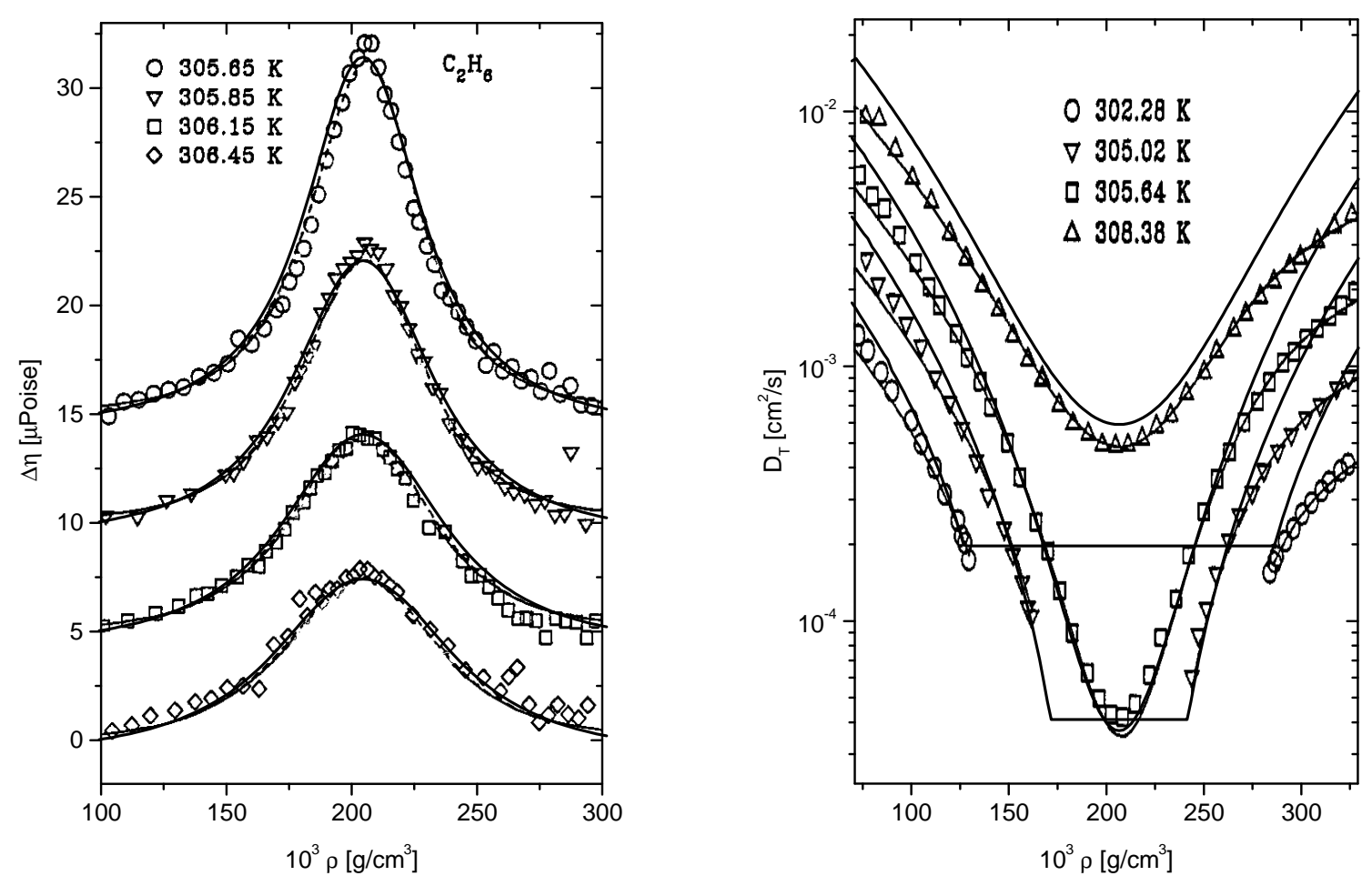

Figure 3. (a) Shear viscosity in $\mathrm{C}_{2} \mathrm{H}_{6}$ along various isotherms. The plot contains our results (thick curves) as well as experimental data [15] and theoretical results of the mode coupling theory (thin dashed curves) [14]. The curves were shifted by 5,10 or $15 \mu$ Poise respectively for better clearness. (b) Thermal diffusivity in $\mathrm{C}_{2} \mathrm{H}_{6}$ along various isotherms. The plot contains our results (thick curves) as well as experimental data [16] and theoretical results of the mode coupling theory (thin curves) [14]. We have used the analytic background expressions of [17] (from $[2])$.

As an example the comparison of a calculation of the shear viscosity and the thermal diffusivity is shown in figure 3. Only the shear viscosity data at $\Delta \rho=0$ are used to determine the nonuniversal parameters. It should be mentioned that an extension of such calculations to sound propagation is possible and it has also been considered for pure fluids in [1] and for mixtures in [3].

\section{Scaling for pure fluids}

The dynamic scaling assumption states that the dynamic correlation function $\chi_{\text {dyn }}$ of the OP in the asymptotic region is a homogeneous function of its variables and can be written in the form (see [13])

$$
\chi_{\mathrm{dyn}}(\xi, k, \omega)=\frac{\chi_{\mathrm{st}}(\xi, k)}{\omega_{\mathrm{c}}(\xi, k)} F\left(\frac{\omega}{\omega_{\mathrm{c}}(\xi, k)}, k \xi\right) .
$$


The characteristic frequency $\omega_{\mathrm{c}}$ (we define the half width at half height) is also a homogeneous function

$$
\omega_{\mathrm{c}}(\xi, k)=k^{z} f(k \xi),
$$

where the frequency is measured in an appropriate time scale. The static correlation function $\chi_{\text {st }}$ also scales as

$$
\chi_{\mathrm{st}}(\xi, k)=k^{-2+\eta} g(k \xi),
$$

and the shape function $F(y, x)\left(y=\omega / \omega_{\mathrm{c}}(\xi, k)\right.$ and $\left.x=k \xi\right)$ fulfils the relations

$$
\int \mathrm{d} y F(y, x)=2 \pi, \quad F(1, x)=\frac{1}{2} F(0, x) .
$$

Since the OP is conserved for the cases considered here, the correlation function has to be proportional to $k^{2}$ in the limit $k \rightarrow 0$. Neglecting static and dynamic interactions of fluctuations, conventional theory leads to the exponents $\eta=0$ and $z=4$ (for a nonconserved OP the exponent would be $z=2$ ). Moreover, the shape function $F(y, x)$ is independent of $x$. RGT calculates the values of the exponents as $\eta \sim 0.04$ and $z=4-\eta-x_{\lambda} \sim 3$ since $x_{\lambda} \sim 0.916$ [8].

In addition to this considerable change of the exponent's values, the shape function $F(y, x)$ will depend on $x$. Let us now consider several regions in the $\left(\xi^{-1}, k, \omega\right)$ space.

In the hydrodynamic region $k \xi \ll 1$ in fluids and mixtures the dynamic behaviour is described by diffusive modes, e.g. $\omega=D k^{2}$ for the thermal diffusion, that means for the temperature dependence of the OP diffusion $D$

$$
D(\xi)=\Gamma(\xi) / \chi_{\mathrm{st}}(\xi) \sim \xi^{2-z}, \quad \Gamma(\xi) \sim \xi^{2-z+\gamma / \nu}
$$

with $\Gamma$ the OP Onsager coefficient. In the conventional theory $\Gamma$ is noncritical, whereas RGT predicts $\Gamma \sim \xi^{x_{\lambda}}$ (we have used the static scaling law $\gamma=\nu(2-\eta)$ ). In the background both $\Gamma, \chi$ and $D$ are temperature independent (apart from a weak analytic temperature dependence outside the scope of our considerations). Throughout the hydrodynamic regime, the shape of the correlation function is of Lorentzian form

$$
\chi_{\text {dyn }}(\xi, k, \omega)=\chi_{\text {st }}(\xi, k) 2 \Re \frac{1}{-\mathrm{i} \omega+D(\xi) k^{2}}
$$

thus

$$
F(y, x<<1)=2 \Re \frac{1}{\mathrm{i} y+1} .
$$

In order to consider the scaling laws in the critical region $k \xi \gg 1$ we may directly go to $T_{\mathrm{c}}$. At $T_{\mathrm{c}}(\xi=\infty)$, the correlation function can be written as

$$
\chi_{\text {dyn }}(k, \omega) \sim k^{-z-2+\eta} F(y, \infty)
$$

since $\omega_{\mathrm{c}} \sim k^{z}$ and $\chi_{\mathrm{st}} \sim k^{-2+\eta}$. Because of the conservation property of the correlation function in the limit $k \rightarrow 0$ mentioned above and because its value is finite in 


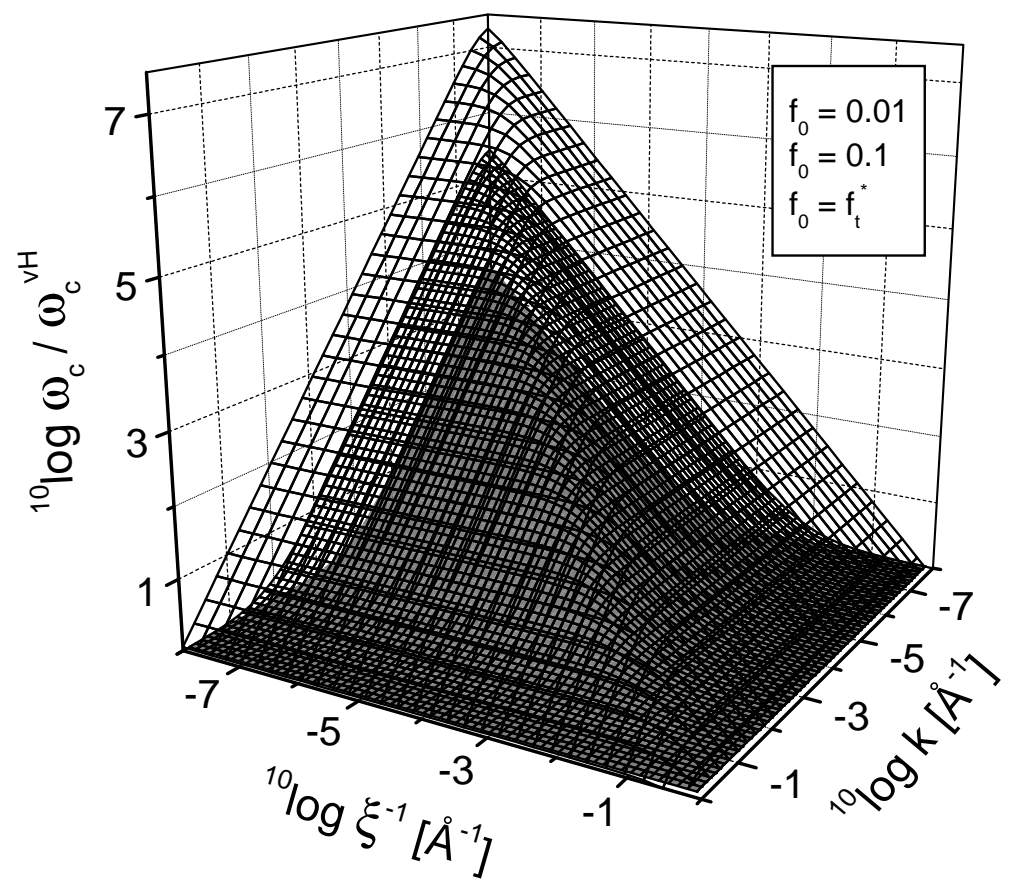

Figure 4. Ratio of the nonasymptotic characteristic frequency of the RG calculation to the characteristic frequency of the van Hove theory for different background values of the mode coupling $f_{0}$. Upper wide mash $f_{0}=f^{*}$, next lower mash $f_{0}=0.1$, inner 'pyramid' $f_{0}=0.01$. For $f_{0}=0$ one obtains for the ratio the bottom plane. The smaller the value of the mode coupling the smaller is the region around the critical point $k=\xi^{-1}=0$ where the asymptotic power laws are seen.

the limit $\omega \rightarrow 0$, the shape function behaves as

$$
\begin{array}{r}
F(y, \infty) \sim \text { const, } \quad y \rightarrow 0 \\
F(y, \infty) \sim y^{-(z+4-\eta) / z}, \quad y \rightarrow \infty
\end{array}
$$

This shows that RGT predicts a non-Lorentzian shape at $T_{\mathrm{c}}$ since it decays faster in the scaled frequency, namely roughly as $y^{-2.3}$ instead of $y^{-2}$. In other words this demonstrates the non Markovian property of the OP time correlations.

\section{Characteristic frequency}

A complete calculation of the shape function and width in RG-theory is lacking. A one loop calculation gives no frequency dependent perturbational contribution to the order parameter vertex functions, which seems to be in contradiction to the scaling arguments of the preceding section. In the following we remain within the Lorentzian approximation for the correlation function, then the result of a nonasymptotic RG calculation of the characteristic frequency yields

$$
\omega_{\mathrm{c}}(k, x)=\Gamma_{\mathrm{as}} k^{z}\left(\frac{1+x^{2}}{x^{2}}\right)^{1-x_{\lambda} / 2} c_{\mathrm{na}}^{x_{\lambda}}(k, x) f(k, x)
$$




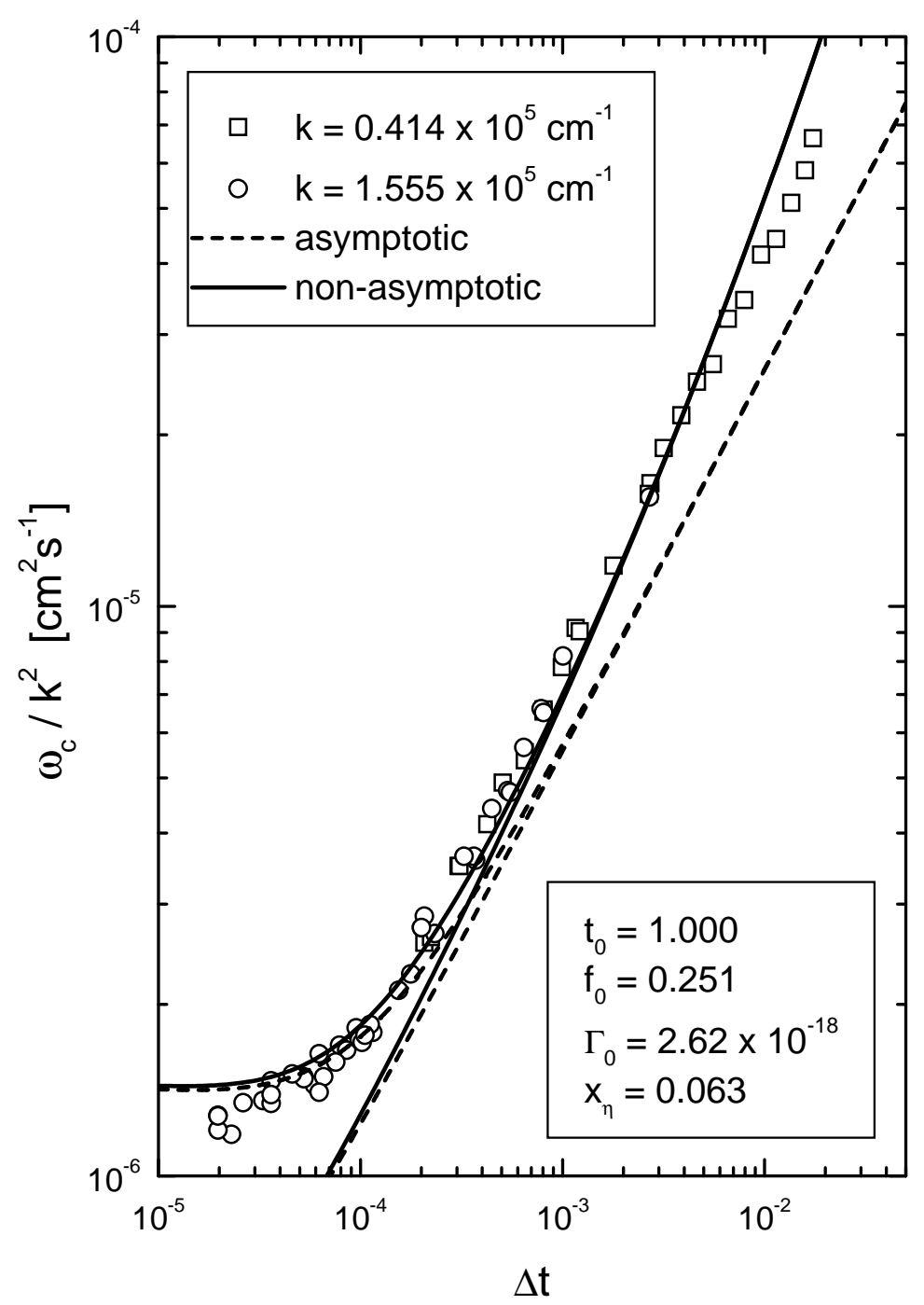

Figure 5. Nonasymptotic and asymptotic characteristic frequency $\omega_{\mathrm{c}}$ as function of temeperature for diffenrent wave vectors $k$ calculated in $\varepsilon$-expansion. Data for $\mathrm{CO}_{2}$ from [18].

with

$$
c_{\mathrm{na}}(k, x)=\left[1+\frac{k}{k_{0}} \sqrt{\frac{1+x^{2}}{x^{2}}}\right]
$$

and

$$
f(k, x)=1-\frac{3}{38 c_{\mathrm{na}}(k, x)}\left[-5+6 x^{-2} \ln \left(1+x^{2}\right)\right]
$$

where

$$
x=k \xi(t), \quad \Gamma_{\text {as }}=\Gamma_{0}\left(\frac{19}{24} \frac{f_{0}^{2} \ell_{0}}{\xi_{0}}\right)^{x_{\lambda}}, \quad k_{0}^{-1}=\left(\frac{24}{19 f_{0}^{2}}-1\right) \frac{\xi_{0}}{\ell_{0}} .
$$

There are two dynamic non universal parameters. One of them, $\Gamma_{0}$, sets the scale of 
the frequency and the other one, the dimensionless mode coupling $f_{0}$, achieves the crossover from the van Hove theory $\left(f_{0}=0\right.$, and $\left.z=4\right)$

$$
\omega_{\mathrm{c}, \mathrm{vH}}=\Gamma_{0} k^{4}\left(\frac{1+x^{2}}{x^{2}}\right)
$$

to the asymptotic theory $\left(f_{0}=f^{*}, z \sim 3\right)$

$$
\omega_{\mathrm{c}, \mathrm{as}}=\Gamma_{0}\left(\frac{\ell_{0}}{\xi_{0}}\right)^{x_{\lambda}} k^{z}\left(\frac{1+x^{2}}{x^{2}}\right)^{1-x_{\lambda} / 2}\left(1-\frac{3}{38}\left[-5+6 x^{-2} \ln \left(1+x^{2}\right)\right]\right) .
$$

For any finite $f_{0}$, a crossover from the van Hove theory in the background to the asymptotics results near the critical point, $\xi \rightarrow \infty$ and $k \rightarrow 0$. In this case the asymptotic amplitude, $\Gamma_{\text {as }}(29)$, of the characteristic frequency depends on the mode coupling $f_{0}$.

We rewrite the nonasymptotic expression for the characteristic frequency in the following form

$$
\omega_{\mathrm{c}}(k, \xi)=\omega_{\mathrm{c}, \mathrm{vH}}(k, \xi)\left(k \xi_{0}\right)^{-x_{\lambda}}\left(\frac{19}{24} f_{0}^{2} \ell_{0}\right)^{x_{\lambda}}\left(\frac{1+x^{2}}{x^{2}}\right)^{-x_{\lambda} / 2} c_{\mathrm{na}}(k, x)^{x_{\lambda}} f(k, x) .
$$

The crossover behaviour for finite mode coupling $f_{0}$ can be seen in figure 4 . Near $T_{\mathrm{c}}$ the asymptotic expression (31) applies whereas in the background van Hove behaviour is reached. Taking the nonuniversal value for $\Gamma_{0}$ from the shear viscosity the value of $f_{0}$ is adjusted to fit the characteristic frequency data. This leads to a value definitive different from the fixed point value as can be seen from figure 5 . Similar results are obtained for xenon [11].

I thank G.Flossmann and G.Moser, the authors of joint papers, for collaboration in this research. The research is supported by the Fonds zur Foerderung der Wissenschaftlichen Forschung under project 12422-TPH.

\section{References}

1. Folk R., Moser G. Frequency-dependent shear viscosity, sound velocity and sound attenuation near the critical point in liquids. I. Theoretical results. // Phys. Rev. E, 1998, vol. 57, No. 1, p. 683-704; II. Comparison with experiment. // Phys. Rev. E, 1998, vol. 57, No. 1, p. 705-719.

2. Flossmann G., Folk R., Moser G. Frequency-dependent shear viscosity, sound velocity and sound attenuation near the critical point in liquids. III. The shear viscosity. // Phys. Rev. E, 1999, vol. 60, No. 1, p. 779-791.

3. Folk R., Moser G. Critical dynamics in mixtures. // Phys. Rev. E, 1998, vol. 58, No. 5, p. 6246-6274; Critical sound propagation in mixtures. // Europhys. Lett., 1998, vol. 41, No. 2, p. 177-182.

4. Bausch R., Janssen H.K., Wagner H. Renormalized field theory of critical dynamics. // Z. Phys. B, 1976, vol. 24, p. 113-127. 
5. Sengers Jan van Effects of critical fluctuations on the thermodynamic and transport properties of supercritical fluids. - In: Supercritical Fluids, ed. Kiran E. and LeveltSengers J.M.H., p. 231-271.

6. Huang C.-C., Ho J.T., Parametric equations of state for fluids near the critical point // Phys. Rev. A, 1973, vol. 7, No. 4, p. 1304-1311.

7. Yuchnovskii I.R. Phase Transitions of the Second Order. Collective Variables Method. Singapure, World Scientific, 1987.

8. Siggia E.D., Halperin B.I., Hohenberg P.C. Renormalization-group treatment of the critical dynamics of the binary-fluid and gas-liquid transition. // Phys. Rev. B, 1976, vol. 13, No. 5, p. 2110-2132.

9. Berg R.F., Moldover M.R., Zimmerli G.A. Frequency-dependent shear viscosity of xenon near the critical point. // Phys. Rev. E, 1999, vol. 60, No. 4, p. 4079-4098.

10. Bhattacharjee J.K., Ferrell R.A. Dynamic scaling for the critical viscosity of a classical fluid. // Phys. Lett., 1980, vol. 76A, No. 3,4, p. 290-292; Frequency-dependent critical viscosity of a classical fluid. // Phys. Rev. A, 1983, vol. 27, p. 1544-1555.

11. Flossmann G., Folk R. Critical light scattering in liquids. // Phys. Rev. E, 2000 (in print).

12. In two loop order one has to calculate, besides the exponent function (which are already known), the amplitude functions of the vertex function for the order parameter and the transverse current. From the zero frequency expressions in the hydrodynamic limit one obtains the two loop value of the Kawasaki ratio (which is expected to be near the one loop value), from the frequency dependence the frequency dependent shear viscosity and from the complete expression for the $\mathrm{OP}$ vertex functions the non Lorentzian shape function and its width. Moser G., Folk R., Flossmann G. (in preparation).

13. Folk R., Moser G. - In: Lectures on Cooperative Phenomena in Condensed Matter Physics. ed. D.I.Uzunov, Sofia, Heron Press, 1996.

14. Luettmer-Strathmann J., Sengers J.V., Olchowy G.A. Non-asymptotic critical behaviour of the transport properties of fluids. // J. Chem. Phys., 1995, vol. 103, No. 17, p. $7482-7500$.

15. Iwasaki H., Takahashi M. Viscosity of carbon dioxide and ethane. // J. Chem. Phys., 1981, vol. 74, No. 3, p. 1930-1943 (data taken from [14]).

16. Jany P., Straub J. Thermal diffusivity of fluids in a broad region around the critical point. // Int. J. Thermophys., 1987, vol. 12, No. 8, p. 165-180 (data taken from [14]).

17. Vesovic V., Wakeham W. A., Luettmer-Strathmann J., Sengers J.V., Millat J., Vogel E., Assael M.J. The transport properties of ethane. II. Thermal conductivity. // Int. J. Thermophys., 1994, vol. 15, No. 1, p. 33-66.

18. Swinney H.L., Henry D.L. Dynamics of fluids near the critical point: decay rate of order-parameter fluctuations. // Phys. Rev. A, 1973, vol. 5, No. 1, p. 2586-2617. 


\section{Критичний перенос і критичне розсіяння у плинах}

\section{Р.Фольк}

Інститут теоретичної фізики, Університет м. Лінца, Австрія

Отримано 1 березня 2000 p.

Розглядаються критичні властивості плинів, що індуковані критичними флюктуаціями параметра порядку. Теорія описує кросовер від аналітичної фонової поведінки до асимптотичної поведінки для кількох динамічних величин.

Ключові слова: фазовий перехід газ-рідина, критична динаміка, розсіяння світла, коефіцієнти переносу

PACS: 05.70.Jk, 64.60.Ht, 64.70.Fx, 64.70.Ja 